\title{
Pharmacovigilance in India: A Need Indeed
}

\author{
Mohammad Arif Khan ${ }^{1 *}$, Krishna Pandey ${ }^{2}$ and Krishnamurti ${ }^{1}$ \\ ${ }^{1}$ Department of Pharmacy Practice, National Institute of Pharmaceutical Education and Research, Hajipur, India \\ ${ }^{2}$ Department of Clinical Medicine, Rajendra Memorial Research Institute of Medical Sciences, Patna, India
}

*Corresponding author: Mohammad Arif Khan, Department of Pharmacy Practice, National Institute of Pharmaceutical Education and Research, Hajipur, India, Tel: +91 9470813689; E-mail: khhan.arif@gmail.com

Rec Date: March 6, 2014, Acc Date: March 10, 2014, Pub Date: March 17, 2014

Copyright: (C) 2014 Khan MA, et al. This is an open-access article distributed under the terms of the Creative Commons Attribution License, which permits unrestricted use, distribution, and reproduction in any medium, provided the original author and source are credited.

\section{Abstract}

Along with the advancement in the field of pharmacovigilance, numerous challenges have emerged with the exponential rise in the incidence of adverse drug reactions resulting in to the increased rates of hospitalization, contributing to the growth of overall burden of disease which is somewhere failing to be managed efficiently, consequently giving space for the emergence of the concept of Vigilo-Pharmacists for the fruition of these lacunas.

\section{Introduction}

The concept of pharmacovigilance to the world is not new and dates back to its origin after the Thalidomide disaster which occurred in the early 1960's, where the drug proved to be a potent teratogen, responsible for about 10,000 cases of phocomelia and amelia (deformed limbs), introduced as a safe sedative and antiemetic. In the early 1970's another drug disaster occurred by a cardioselective betablocker- Proctalol, introduced for the treatment of angina and hypertension, resulted into oculo-mucocutaneous syndrome. Later on in the early 1980's several deaths were reported as a result of renal and hepatic failure as a consequence of the use of Benaxoprofen which was a NSAID (Non Steroidal Anti- Inflammatory Drug).

Despite of the rapid and continuous development in the field of pharmacovigilance, the incidence of ADRs (Adverse Drug Reactions) and ADEs (Adverse Drug Events) have increased considerably and are still multiplying with the exponentially growing rate of drug users across over the globe. Statistics and synthesised pooling of results from meta-analysis suggests that around $6.5-7 \%$ of the hospital admissions are attributable to ADRs whereas in the United States every fourth to fifth death is somehow due to ADRs and is supposed to increase with the passage of time.

Giving due respect to the evident fact that 'No drug which is pharmacologically active is without hazards' becomes quite obvious on their usage to have ADRs which can be defined as "any unintended or noxious effect that is attributable to a medicine when it has been given within the normal range of doses used in man", but at the same time should not be confused with the similar sounding terms, side effects and ADEs where the former is defined as "any unintended effect of medicine" which under normal circumstances is undesirable but could be beneficial". For example; the anxiety reducing effect of a betablocker in an anxious hypertensive. The latter (ADEs) can be defined as any undesirable occurrence that occurs during the course of treatment which may or may not have a causal link with the drug. Here a big question which arises is that how these ADRs and ADRs can be identified and differentiated from each other? Need of a skilled health care professional? Also the implementation of specific terms, ADRs, suspected ADRs and ADEs is not as easier as it appears and is quite incomprehensive to differentiate among them. Need of skilled healthcare professionals?

\section{Pharmacovigilance in India}

These concepts of pharmacovigilance gets trivial or almost seem inefficient to serve the purpose especially in the developing countries like India with a population of teaming millions, low literacy rate and high rate of illness. Thus it is quite obvious that the overall burden of treatment is over to the clinicians whose primary focus of concern is the disease management, consequently $\mathrm{ADR}$ reporting becomes secondary one, which is apparently the main cause of underreporting for $90 \%$ of the cases, therefore cannot contribute much as they are supposed to or required as, for the clinical data management of the pharmacovigilance programme. Need of skilled healthcare professionals? Most of the ADRs which can reported are either retrieved through the patient's interview or by their regular and close monitoring through phone contact or by regular laboratory procedures which is apparently not exactly possible due to lack of or bound timeframe. Thus, the ADRs or ADEs whether suspected or definite is either not reported or sometimes neglected. Need of skilled healthcare professionals?

\section{Need of Vigilo-Pharmacists}

Keeping an eye over the few above mentioned facts, it becomes quite evident that a great necessity is felt for the concept of "VigiloPharmacist" to be an established fact, which is as discussed earlier through 'Need of skilled healthcare professionals?'

World Health Organization defines 'Pharmacovigilance' as the science or activities relating to the detection, assessment, understanding and prevention of adverse effects or any other drug related problem. The first and the foremost segment of importance is the 'detection' which is not as easier as it appears to be and its further classification into ADR or ADE. Once detected can be assessed and may be prevented. Detection could be easier by spontaneous ADR reporting methodology but underreporting is a serious threat for the failure of 'Signal Detection' or early warning of the manifestation. Vigilo-Pharmacists can play a vital role here by managing or pretty much preventing the threat of underreporting, through patient 
Citation: Mohammad Arif Khan, Krishna Pandey, Krishnamurthi (2014) Pharmacovigilance in India: A Need Indeed. J Pharmacovigilance 2: 126. doi:10.4172/2329-6887.1000126

Page 2 of 2

interview and their regular monitoring, thereby contributing efficiently to the GPP (Good Pharmacovigilance Practice). There are ADRs which are preventable and can be prevented by close monitoring of the patient as for example; Agranulocytosis (decrease in WBC count) which is a classical ADR attributable to an atypical antipsychotic: Closapine. Closer monitoring of the WBCs in patients taking closapine can prevent the causation of agranulocytosis, saving the patient from fatal consequences, improving the practice of pharmacovigilance, contributing to the practice of GPP.

Thus, it evident enough to conclude that there is plenty of space at the bottom, that can be brought to fruition by the implementation of skilled healthcare professionals; the so called 'Vigilo-Pharmacists' to advance the practice of pharmacovigilance. 